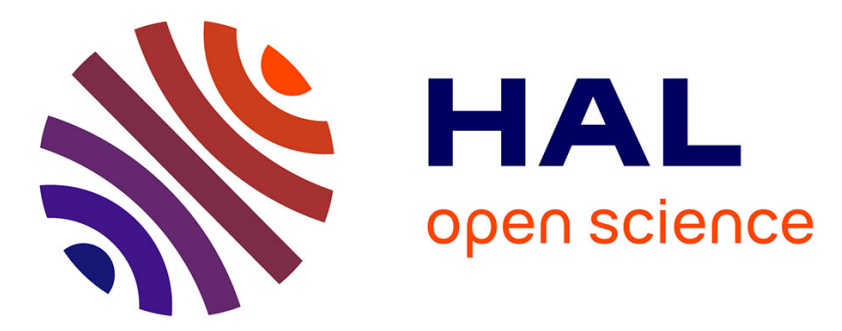

\title{
Dispositif d'intégration utilisant un phénomène d'origine thermique. Application à l'étude de la stabilisation des fluctuations d'un champ magnétique par effet d'induction
}

G. Raoult, R. Fanguin, F. Blanc

\section{To cite this version:}

G. Raoult, R. Fanguin, F. Blanc. Dispositif d'intégration utilisant un phénomène d'origine thermique. Application à l'étude de la stabilisation des fluctuations d'un champ magnétique par effet d'induction. Revue de Physique Appliquée, 1967, 2 (4), pp.292-300. 10.1051/rphysap:0196700204029200 . jpa00242808

\section{HAL Id: jpa-00242808 https://hal.science/jpa-00242808}

Submitted on 1 Jan 1967

HAL is a multi-disciplinary open access archive for the deposit and dissemination of scientific research documents, whether they are published or not. The documents may come from teaching and research institutions in France or abroad, or from public or private research centers.
L'archive ouverte pluridisciplinaire HAL, est destinée au dépôt et à la diffusion de documents scientifiques de niveau recherche, publiés ou non, émanant des établissements d'enseignement et de recherche français ou étrangers, des laboratoires publics ou privés. 


\title{
DISPOSITIF D'INTÉGRATION UTILISANT UN PHÉNOMÈNE D'ORIGINE THERMIQUE APPLICATION A L'ÉTUDE DE LA STABILISATION DES FLUGTUATIONS D'UN GHAMP MAGNÉTIQUE PAR EFFET D'INDUGTION
}

\author{
Par G. RAOULT, R. FANGUIN et F. BLANC, \\ Laboratoire de Radioélectricité de la Faculté des Sciences de Clermont-Ferrand.
}

\begin{abstract}
Résumé. - Il est souvent nécessaire d'intégrer par rapport au temps un signal lentement variable. Dans la plupart des cas, l'intégration des signaux rapides s'effectue assez simplement; il n'en est pas de même pour des signaux très lents pouvant aller jusqu'à $0,005 \mathrm{~Hz}$. Nous proposons un dispositif simple et irréversible qui utilise la variation de température produite par le signal sur un échantillon possédant une inertie calorifique convenable. On montre que, dans ces conditions, la température représente à une constante près l'intégrale du signal initial. Un tel intégrateur a été utilisé dans la réalisation d'un stabilisateur de flux par effet d'induction pour obtenir un champ magnétique stable à mieux que $10^{-5}$ près, sauf pour des dérives très lentes. Dans ce cas particulier, on a utilisé comme échantillon le filament d'une petite lampe à incandescence dont on utilise le rayonnement. Le flux lumineux est reçu par une photodiode qui transmet l'information sous forme de courant au système correcteur. Nous avons étudié l'asservissement et remarqué que dans l'entrefer de l'électroaimant on pouvait obtenir une meilleure stabilisation du flux que celle mesurée au niveau des bobines de lecture. Il semble qu'une très faible concentration des lignes d'induction dans l'entrefer due aux bobines de correction puisse expliquer ce résultat.
\end{abstract}

Abstract. - It is often necessary to integrate a signal slowly varying with time. In most cases, integration of rapid signals can be performed easily, it is not the same for very slow signals which can be as slow as $0.005 \mathrm{Hertz}$. We propose a very simple and irreversible system, which uses the variation of temperature of a sample. One can show that this temperature is, to a multiplicative constant, the integral of the signal. A theoretical study of the system is made. Such an integrator has been used for stabilizing the field of an electromagnet, with a precision better than $10^{-5}$ except for very slow variations. In this case, the sample was the filament of an incandescent lamp, the light of which is received by a photodiode which transmits the information as a current to the correcting system. We have studied the stabilizing loop and observed that the flux stabilization is better in the center of the field than it is at the reading coils. This seems to be due to a certain concentration of flux lines in the gap.

\section{A. DISPOSITIF D'INTEGRATION UTIUISANT UN PHENOMENE D'ORIGINE THERMIQUE}

I. Introduction. - De nombreux problèmes physiques conduisent à obtenir la primitive d'un signal électrique donné. On connaît le dispositif classique d'intégration par simple condensateur ou celui plus élaboré de l'intégrateur de Miller qui permet d'opérer aux très basses fréquences. Les difficultés (fuites des condensateurs, complexité des dispositifs électroniques...) deviennent d'autant plus importantes que les phénomènes à intégrer sont plus lents. Nous proposons un dispositif irréversible (entrée et sortie totalement découplées) très simple qui permet l'intégration d'un signal jusqu'aux très basses fréquences.
II. Principe. - On considère un corps possédant une capacité calorifique $M c$. On lui fournit sous forme thermique une énergie dépendant du phénomène à intégrer et qui lui sera proportionnelle. On détecte ensuite la variation de température de l'échantillon à l'aide d'un élément soit thermosensible, soit sensible au rayonnement. Le signal obtenu est intégré par rapport au signal initial.

Les données ont leurs symboles usuels : nous appellerons, en notant toujours les quantités de chaleur en unités mécaniques, $\mathrm{d} Q, \mathrm{~d} Q_{1}, \mathrm{~d} Q_{2}, \mathrm{~d} Q_{3}$ les quantités de chaleur fournie, absorbée, perdue par rayonnement, perdue par conduction ou convection à et par l'échantillon pendant le temps $\mathrm{d} t . W_{0}$ et $W_{\mathrm{a}}(t)$ sont les puissances constante et fournie par le signal; $T_{0}$ est la température obtenue par $W_{0}, T_{\mathrm{a}}(t)$ la variation de température provoquée par le signal, 
$T_{\mathrm{A}}$ la température ambiante, $K$ le coefficient de refroidissement de l'échantillon :

$$
\begin{aligned}
& \left\{\begin{array}{l}
\mathrm{d} Q=\left[W_{\mathrm{a}}(t)+W_{0}\right] \mathrm{d} t \\
\mathrm{~d} Q_{1}=M c \mathrm{~d} T \\
\mathrm{~d} Q_{2}=\alpha \sigma S\left(T^{4}-T_{\mathrm{A}}^{4}\right) \mathrm{d} t \\
\mathrm{~d} Q_{3}=K\left(T-T_{\mathrm{A}}\right) \mathrm{d} t
\end{array}\right. \\
& \mathrm{d} Q=\mathrm{d} Q_{1}+\mathrm{d} Q_{2}+\mathrm{d} Q_{3}
\end{aligned}
$$

c'est-à-dire :

$$
\begin{aligned}
& {\left[W_{0}+W_{\mathrm{a}}(t)\right] \mathrm{d} t} \\
& \quad=M c \mathrm{~d} T+\alpha \sigma S\left(T^{4}-T_{\mathrm{A}}^{4}\right) \mathrm{d} t+K\left(T-T_{\mathrm{A}}\right) \mathrm{d} t .
\end{aligned}
$$

Nous supposons que $W_{\mathrm{a}}(t)$ est petit devant $W_{0}$; $T_{\mathrm{a}}(t)$ est très petit devant $T_{0}$, et $T^{4} \simeq T_{0}^{4}+4 T_{0}^{3} T_{\mathrm{a}}(t)$, nous aurons :

$$
\begin{aligned}
M c \frac{\mathrm{d} T_{\mathrm{a}}(t)}{\mathrm{d} t}+ & \left(K+4 \alpha \sigma S T_{0}^{3}\right) T_{\mathrm{a}}(t)-W_{\mathrm{a}}(t) \\
& =W_{0}-K\left(T_{0}-T_{\mathrm{A}}\right)-\alpha \sigma S\left(T_{0}^{4}-T_{\mathrm{A}}^{4}\right)
\end{aligned}
$$

si $W_{\mathrm{a}}(t)=0, T_{\mathrm{a}}(t)=0, \mathrm{~d} T_{\mathrm{a}}(t) / \mathrm{d} t=0$ et le premier membre est nul, on a donc :

$$
W_{0}-K\left(T_{0}-T_{\mathrm{A}}\right)-\alpha \sigma S\left(T_{0}^{4}-T_{\mathrm{A}}^{4}\right)=0
$$

qui permet de calculer $K$ en fonction de $W_{0}, T_{0}$ et des constantes physiques.

L'équation se réduit donc à :

$$
M c \frac{\mathrm{d} T_{\mathrm{a}}(t)}{\mathrm{d} t}+\left(K+4 \alpha \sigma S T_{0}^{3}\right) T_{\mathrm{a}}(t)-W_{\mathrm{a}}(t)=0
$$

si nous posons que $W_{\mathrm{a}}(t)$ est de la forme

$$
W_{\mathrm{a}}(t)=W_{\mathrm{m}} \mathrm{e}^{j \omega t}
$$

$T_{\mathrm{a}}$ sera :

$$
T_{\mathrm{a}}(t)=T_{\mathrm{m}} \mathrm{e}^{j(\omega t+\varphi)}
$$

et on en déduit :

$$
\begin{array}{lr}
\operatorname{tg} \varphi=-\frac{M c \omega}{K+4 \alpha \sigma S T_{0}^{3}} & \varphi \simeq \\
T_{\mathrm{m}}=\frac{W_{\mathrm{m}}}{\sqrt{M^{2} c^{2} \omega^{2}+\left(K+4 \alpha \sigma S T_{0}^{3}\right)^{2}}} \simeq \frac{W_{\mathrm{m}}}{M c \omega} .
\end{array}
$$$$
\varphi \simeq 90^{\circ}
$$

On voit que si $M c \omega$ est grand par rapport au terme $\left(K+4 \alpha \sigma S T_{0}^{3}\right)$ (condition $\left.\mathrm{B}\right)$, ce qui revient à négliger le terme en $T_{\mathrm{a}}(t)$ dans l'équation $(\mathrm{A})$ et à écrire :

$$
T_{\mathrm{a}}(t)=\frac{1}{M c} \int W_{\mathrm{a}}(t) \mathrm{d} t
$$

la variation de température $T_{\mathrm{a}}(t)$ est à un coefficient de proportionnalité près l'intégrale du signal $W_{\mathrm{a}}(t)$.

Étant donné la linéarité de l'équation (A), on peut étendre les résultats précédents à des signaux de forme plus compliquée pouvant être décomposés en série de Fourier, à condition de respecter la condition $\mathrm{B}$. Cette dernière est d'autant mieux vérifiée que la fréquence est plus élevée.
III. Exemples de réalisations et résultats. - Les exemples diffèrent en général en fonction de la nature de l'élément sensible employé et de l'échantillon choisi.

a) Dispositif utilisant le rayonnement lumineux d'une lampe à incandescence. - Nous avons réalisé deux expériences, l'une avec un filament de faible capacité calorifique, l'autre avec un filament de capacité calorifique nettement supérieure. Dans les deux cas, on a utilisé comme élément sensible une photodiode PHG 1 de la Cosem.

Le montage utilisé était le suivant : Un générateur basse fréquence, donnant des signaux rectangulaires symétriques, commande un transistor de puissance qui alimente la lampe à incandescence. Cette dernière éclaire la photodiode PHG 1 et l'ensemble lampephotodiode est placé dans une enceinte étanche à la lumière afin d'éviter les perturbations d'origine extérieure. La photodiode est alimentée par une pile de 27 volts en série avec une résistance de charge de $10000 \Omega$.

Un oscillographe très basse fréquence à double trace, muni d'un dispositif photographique, permet d'enregistrer simultanément le signal rectangulaire appliqué sur la lampe et le signal obtenu sur la résistance de charge de la photodiode.

1) Résultats obtenus avec la lampe de faible capacité calorifique. - Nous avons utilisé une lampe lentille de $2,25 \mathrm{~V}$ et $0,25 \mathrm{~A}$, dont nous avons mesuré les autres caractéristiques qui nous ont donné :

$$
K \simeq 10^{-4} \mathrm{~W} / \text { degré d'où } \operatorname{tg} \varphi=0,44 f .
$$

Ci-joint une série de photographies pour les fréquences $50-20-5-2 \mathrm{~Hz}$. Nous voyons qu'à $2 \mathrm{~Hz}$ nous n'avons pas encore atteint le palier de l'exponentielle (fig. 1).

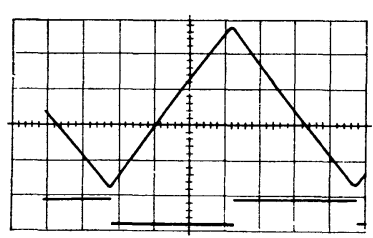

$50 \mathrm{~Hz}$

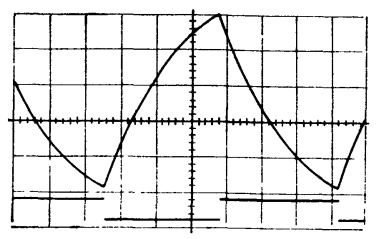

$5 \mathrm{~Hz}$

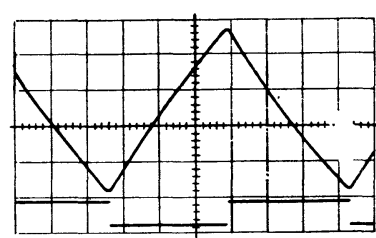

$20 \mathrm{~Hz}$

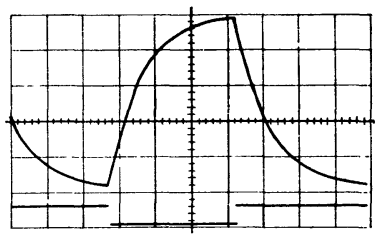

$2 \mathrm{~Hz}$
FIG. 1. - Lampe à faible capacité calorifique. 
2) Résultats obtenus avec la lampe à capacité calorifique plus élevée. - Nous avons utilisé une lampe du type éclairage automobile prévue pour une tension d'alimentation de $6 \mathrm{~V}$ et $3,5 \mathrm{~A}$.

Nous avons calculé la valeur de $K$ :

$$
K \simeq 29,5 \times 10^{-4} \text { d'où } \operatorname{tg} \varphi=4,04 f
$$

ce qui nous donne, en comparant avec l'exemple précédent, la même tg $\varphi$ pour une fréquence environ 10 fois plus faible.

Nous avons effectué une série de photographies pour les fréquences $5-2-0,5-0,2 \mathrm{~Hz}$ (fig. 2); par comparaison, nous remarquons que l'on a gagné approximativement un facteur 10.

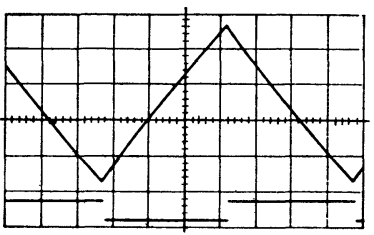

$5 \mathrm{~Hz}$

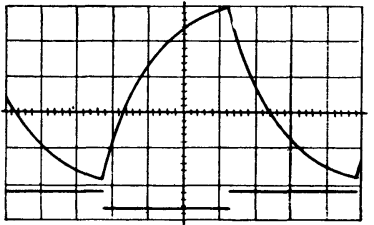

$0,5 \mathrm{~Hz}$

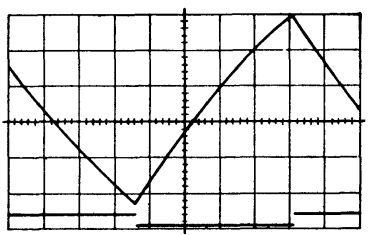

$2 \mathrm{~Hz}$

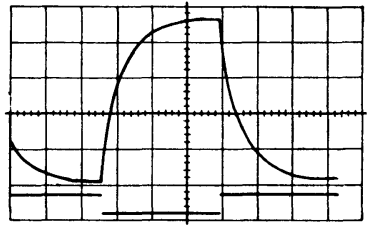

$0,2 \mathrm{~Hz}$
Fig. 2. - Lampe à capacité calorifique élevée.

Avec les dispositifs à lampe à incandescence, on ne peut augmenter notablement la capacité calorifique du filament qu'en augmentant aussi la puissance consommée, et à partir d'un certain moment cette puissance devient prohibitive; il est alors nécessaire de chercher un système plus approprié.

b) Dispositif à transistor. - Si l'on veut obtenir une intégration correcte à des fréquences encore plus basses, il faut augmenter la capacité calorifique de l'échantillon.

Dans le but de vérifier expérimentalement si un tel dispositif permettait une intégration correcte à des fréquences très basses, nous avons simplement pris deux transistors Raythéon Y 633 que nous avons mis en contact thermique par la surface plane de leur boîtier à l'aide d'une peinture à l'argent. L'ensemble est placé dans une enceinte à l'abri des perturbations thermiques extérieures. L'un d'eux joue le rôle d'échantillon, l'autre de détecteur thermique.

Nous envoyons un signal rectangulaire symétrique sur la base d'un des transistors réglé à un courant collecteur moyen de l'ordre de $5 \mathrm{~mA}$ pour une tension collecteur-émetteur de 12 volts. Le deuxième tran- sistor servant d'élément thermosensible fonctionne sous son courant résiduel en montage émetteur commun. Ce transistor est alimenté sous 12 volts à travers une résistance de charge aux bornes de laquelle on observe le signal intégré (fig. 3).

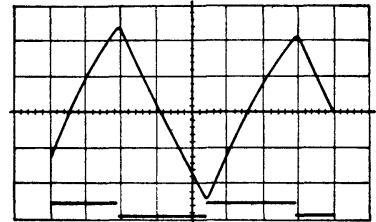

FIG. 3. - Signal initial rectangulaire symétrique par rapport au zéro. Période $0,005 \mathrm{~Hz}$. Enregistrement du signal intégré.

Il est évident que l'on peut remplacer le premier transistor servant d'échantillon par tout autre élément dissipant de l'énergie. On peut en particulier prendre une simple résistance.

\section{B. APPLICATION A L'ETUDE \\ DE LA STABILISATION DES FLUCTUATIONS D'UN CHAMP MAGNETIQUE}

I. L'électroaimant. - Une étude [1], [2] a permis d'améliorer l'homogénéité du champ dans l'entrefer en taillant l'extrémité de la pièce annulaire en forme de tronc de cône ( fig. 4).

La caractéristique induction-courant est linéaire et possède une pente de $8,4 \times 10^{-2}$ tesla/ampère. L'intensité maximum supportée par les enroulements

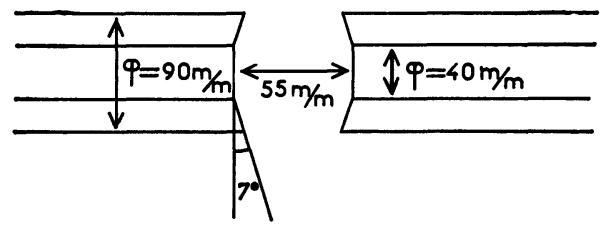

FIG. 4. - Entrefer de l'électroaimant.

magnétisants est de 8,5 ampères, ce qui correspond à un champ magnétique maximum de 0,71 tesla. La résistance des deux enroulements est de $11,5 \Omega$ et leur self de $1,7 \mathrm{H}$ à $50 \mathrm{~Hz}$.

Avec l'alimentation du Laboratoire, on peut compter sur une stabilité à court terme du courant magnétisant de l'ordre de $10^{-4}$. C'est dans le but d'améliorer encore cette stabilité que nous avons réalisé un système à induction d'un type analogue à celui utilisé par Fric dans sa thèse [3].

II. Les différents organes de la chaîne. - a) Les bobines de lecture et de correction. - Les premières, comme les secondes, sont constituées de deux enroulements placés symétriquement sur les pôles de l'électroaimant de part et d'autre de l'entrefer. Les 


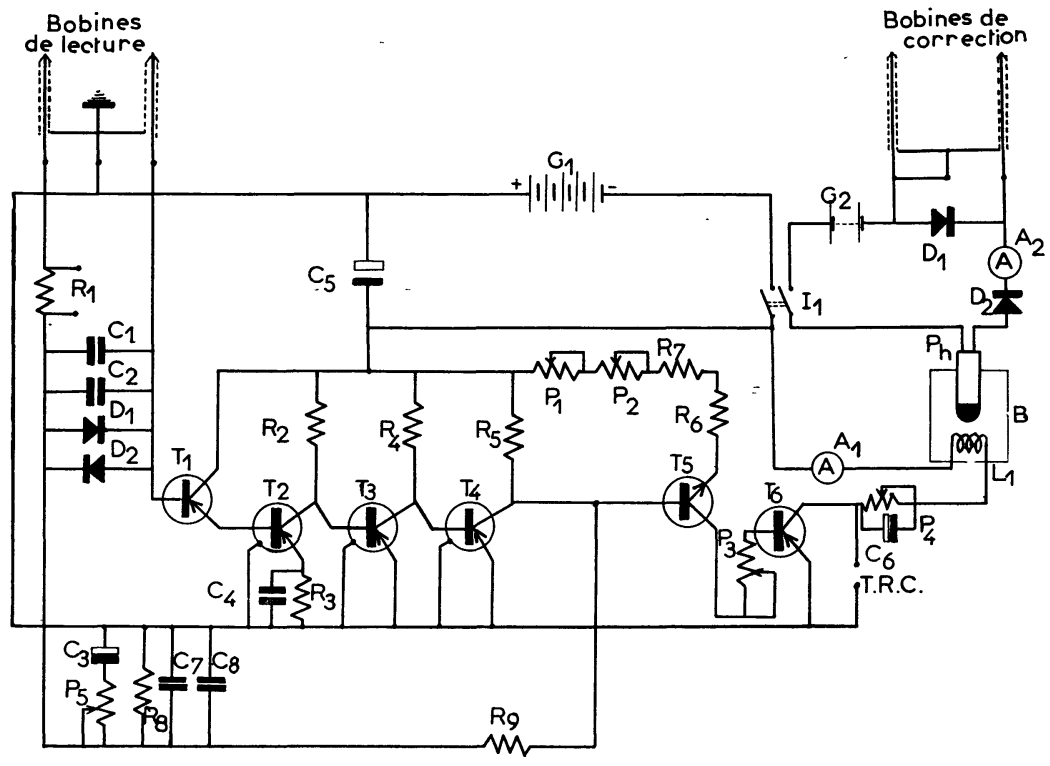

FIG. 5. - Schéma du dispositif d'asservissement.

caractéristiques électriques de deux bobines en série sont :

Pour la lecture : $R=5900 \Omega ; L=19 \mathrm{H}$; capacité répartie $=27 \mathrm{pF}$; fréquence propre de résonance $7000 \mathrm{~Hz}$,

et pour la correction : $R^{\prime}=11000 \Omega ; L^{\prime}=80 \mathrm{H}$; capacité répartie $C^{\prime}=37 \mathrm{pF}$; fréquence propre de résonance $2900 \mathrm{~Hz}$; sensibilité $1,5 \times 10^{-4}$ tesla/mA.

Un système mécanique permet de régler leur position sur l'axe de manière à avoir la meilleure correction possible.

b) Le dispositif électronique d'asservissement (fig. 5). L'amplificateur comprend un amplificateur de tension suivi d'un amplificateur de puissance. Un circuit de contre-réaction assure la stabilité thermique de l'ensemble, tandis qu'une résistance réglable de $1000 \Omega$ en série avec un condensateur de $25000 \mu \mathrm{F}$ règle le gain de manière à garantir la stabilité de fonctionnement.

Deux transistors en liaison directe constituent l'amplificateur de puissance qui alimente une lampe à incandescence du type loupe de 2,25 V-0,25 A.

En l'absence de signal, la lampe est traversée par un courant de repos ajustable à la valeur de $180 \mathrm{~mA}$ par un potentiomètre de $5000 \Omega$. Un milliampèremètre en série dans le circuit permet d'en contrôler la valeur.

La pente de tout l'ensemble a été mesurée et trouvée égale à $4000 \mathrm{~mA} / \mathrm{V}$ à $50 \mathrm{~Hz}$.

L'intégrateur et le circuit de correction sont simplement formés par la lampe à incandescence de faible capacité calorifique dont nous avons donné précédemment les caractéristiques dans l'étude générale et par une photodiode PHG 1. Cet ensemble est placé dans une enceinte fermée à l'abri des perturbations dues aux rayonnements extérieurs. Une pile de $27 \mathrm{~V}$ alimente la photodiode et les bobines de correction. Deux diodes assurent la protection du système contre les surtensions dues aux bobines de correction, qui pourraient naître lors d'une rupture imprévue du courant d'alimentation de l'électroaimant. Un milliampèremètre assure le contrôle du courant photodiode. Un des avantages importants de ce système d'alimentation des bobines de correction par photodiode consiste en l'absence presque totaie de bruit de fond.

III. Etude générale du servomécanisme. - a) Schéma équivalent au circuit d'entrée. - Le circuit est équivalent à celui de la figure 6 :

- Les bobines de lecture dans lesquelles est induite la f.e.m. due à la variation de flux à corriger ont une inductance $L$, leur résistance est $R$;

- La résistance d'entrée de l'amplificateur : $R_{\mathrm{a}}$;

- Les capacités réparties des bobines de lecture et de la capacité d'entrée de l'amplificateur : $C$;

- Les équations partielles permettent d'écrire :

$$
\begin{aligned}
& E_{0}(t)=R\left[C \frac{\mathrm{d} E_{\mathrm{a}}(t)}{\mathrm{d} t}+\frac{E_{\mathrm{a}}(t)}{R_{\mathrm{a}}}\right] \\
& \quad+L\left[C \frac{\mathrm{d}^{2} E_{\mathrm{a}}(t)}{\mathrm{d} t^{2}}+\frac{1}{R_{\mathrm{a}}} \frac{\mathrm{d} E_{\mathrm{a}}(t)}{\mathrm{d} t}\right]+E_{\mathrm{a}}(t) .
\end{aligned}
$$

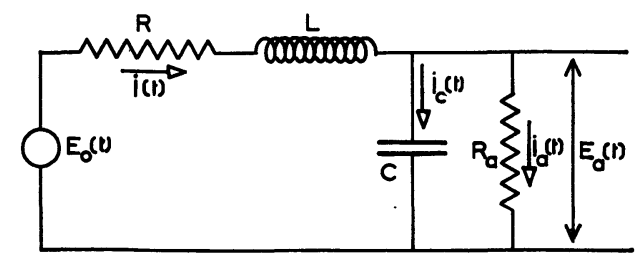

Fig. 6. - Schéma équivalent à l'amplificateur. 
Lorsque $E_{0}(t)$ n'est pas une fonction sinusoïdale du temps, on peut résoudre cette équation par le calcul symbolique à l'aide des transformées de Laplace. Elle s'écrit :

$$
E_{0}=\left[L C p^{2}+\left(R C+\frac{L}{R_{\mathrm{a}}}\right) p+1+\frac{R}{R_{\mathrm{a}}}\right] E_{\mathrm{a}} .
$$

Définissons les trois constantes de temps suivantes :

$$
\sqrt{L C}=\tau_{0}, \quad C R_{\mathrm{a}}=\tau_{1}, \quad \frac{L}{R}=\tau_{2}
$$

et remplaçons $E_{0}$ par $-N p \Phi_{0}$ qui est la traduction symbolique de $E_{0}=-N \frac{\mathrm{d} \Phi_{0}}{\mathrm{~d} t}$, il vient :

$$
E_{\mathrm{a}}=-\frac{N p \Phi_{0}}{\tau_{0}^{2}} \cdot \frac{1}{p^{2}+\left(\frac{1}{\tau_{1}}+\frac{1}{\tau_{2}}\right) p+\frac{1}{\tau_{0}^{2}}+\frac{1}{\tau_{1} \tau_{2}}}
$$

De même, nous avons :

$$
i=\frac{1}{R_{\mathrm{a}}}\left(\tau_{1} p+1\right) E_{\mathrm{a}} .
$$

Appelons $\varphi(p)$ la fonction :

$$
\frac{-N p\left(\tau_{1} p+1\right)}{R_{\mathrm{a}} \tau_{0}^{2}\left[p^{2}+\left(\frac{1}{\tau_{1}}+\frac{1}{\tau_{2}}\right) p+\frac{1}{\tau_{0}^{2}}+\frac{1}{\tau_{1} \tau_{2}}\right]}
$$

Dans ces conditions :

$$
i=\varphi(p) \Phi_{0} \text {. }
$$

La tension $E_{\mathrm{a}}$ précédente commande un amplificateur dont la pente est $G$, le courant de sortie est $I_{\mathrm{a}}=G E_{\mathrm{a}}$ (3) qui alimente l'intégrateur.

b) Étude du dispositif intégrateur [4]. - Ce dispositif est constitué par la lampe et la photodiode, comme nous l'avons indiqué dans l'exposé général. Le courant $I_{\mathrm{a}}$ étant faible, nous pourrons écrire :

$$
W_{\mathrm{a}}(t)=2 \rho I_{0} I_{\mathrm{a}}(t)
$$

l'équation générale $(\mathrm{A})$ est alors en notation symbolique :

$$
M c p T_{\mathrm{a}}+\left(K+4 \alpha \sigma S T_{0}^{3}\right) T_{\mathrm{a}}-2 \rho I_{0} I_{\mathrm{a}}=0
$$

d'où :

$$
T_{\mathrm{a}}=\frac{2 \rho I_{0} I_{\mathrm{a}}}{M c p+\left(K+4 \alpha \sigma S T_{0}^{3}\right)}
$$

ou encore :

$$
T_{\mathrm{a}}=\frac{2 \rho I_{0} I_{\mathrm{a}}}{M c p}\left[\frac{1}{1+\frac{K+4 \alpha \sigma S T_{0}^{3}}{M c p}}\right]
$$

si le terme $\frac{K+4 \alpha \sigma S T_{0}^{3}}{M c p}=H \frac{\omega}{p}$ est petit devant un, nous avons :

$$
T_{\mathrm{a}}=\frac{2 \rho I_{0}}{M c} \cdot \frac{I_{\mathrm{a}}}{p}
$$

qui exprime le fait que la température $T_{\mathrm{a}}$ représente l'intégrale du signal $I_{\mathrm{a}}$.

Si le terme $H$ n'est plus négligeable, mais seulement petit, par rapport à 1 , nous devons revenir à l'équation initiale, et en régime sinusoïdal, nous avons :

$$
T_{\mathrm{a}}(t)=\frac{2 \rho I_{0} I_{\mathrm{a}}(t)}{M c \omega(H+j)} .
$$

La perturbation d'amplitude est du second ordre et vaut : $1 / 2 H^{2}$.

L'erreur sur la phase, par rapport à une intégration parfaite, est alors donnée par :

$$
\operatorname{cotg} \varphi=H
$$

la qualité de l'intégration dépendra donc directement de la petitesse du terme $H$.

c) Circuit de correction. - L'étude de la caractéristique de la photodiode montre qu'elle possède une très grande résistance dynamique, que nous avons pu estimer à une valeur comprise entre 5 et 10 mégohms. Elle se comporte donc comme un injecteur de courant.

Ce fait est très important, car il nous permet de négliger l'impédance des bobines de correction devant celle de la photodiode, ce qui nous autorise à dire que le courant débité par la photodiode est toujours en phase avec $T_{a}$, quelles que soient les caractéristiques des bobines de correction. Nous avons d'ailleurs vérifié expérimentalement ce fait.

Nous avons alors :

$$
i_{\mathrm{s}}=\beta . T_{\mathrm{a}}
$$

$\beta$ étant une constante.

Ce courant $i_{\mathrm{s}}$ se retrouve intégralement dans les bobines de correction à condition toutefois de négliger l'influence de leurs capacités parasites; sinon $i_{\mathrm{s}}$ se répartit entre la self et la capacité parasite. Pour plus de généralité, nous nous placerons dans ce dernier cas.

On fera remarquer cependant que, dans le cas précis de nos bobines et dans un domaine de fréquences où les pertes dans le fer ne sont pas trop grandes $(f<500 \mathrm{~Hz})$, il est tout à fait légitime de négliger les capacités parasites.

Nous avons :

$$
i_{\mathrm{L}^{\prime}}=\frac{i_{\mathrm{s}}}{1+C^{\prime} p\left(L^{\prime} p+R^{\prime}\right)}
$$

posons $\sqrt{L^{\prime} C^{\prime}}=\tau_{0}^{\prime}, \quad C^{\prime} R^{\prime}=\tau^{\prime}$ :

$$
i_{\mathrm{L}^{\prime}}=\frac{i_{\mathrm{s}}}{\tau_{0}^{\prime 2} p^{2}+\tau^{\prime} p+1}
$$


on peut justifier l'hypothèse $i_{\mathrm{L}^{\prime}} \simeq i_{\mathrm{s}}$ pour les fréquences inférieures à $500 \mathrm{~Hz}$. On trouve en effet dans notre cas, pour $500 \mathrm{~Hz}$ :

$$
I_{L^{\prime}}=1,03 I_{\mathrm{s}} ; \quad \operatorname{tg} \varphi^{\prime}=-0,001 .
$$

Il est à noter que l'emploi de la photodiode élimine, $\mathrm{du}$ fait de sa très grande résistance dynamique, d'une part, et de la liaison irréversible photodiode-lampe, d'autre part, toute réaction des bobines de correction sur le stabilisateur. En particulier, les variations de flux qui induisent une f.e.m. dans les bobines de correction ne peuvent donner naissance à un courant que si ce dernier se referme sur la capacité parasite. Ges courants sont négligeables loin de la résonance.

d) Fonction de transfert de la chaîne en boucle ouverte. Pour obtenir la fonction de transfert de la chaîne d'action [6], il suffit de rassembler les résultats (1), (3), (4), (5) et (6) obtenus dans ce qui précède. On aura alors la relation qui lie $i_{L^{\prime}}$ au flux $\Phi_{0}$ traversant les bobines de lecture :

$$
i_{\mathrm{L}^{\prime}}=-\frac{2 \beta G_{\rho} I_{0} N}{M c \tau_{0}^{2}}\left(\frac{1}{\tau_{0}^{\prime 2} p^{2}+\tau_{p}^{\prime}+1}\right)\left(\frac{1}{1+\frac{K+4 \alpha \sigma S T_{0}^{3}}{M c p}}\right)\left(\frac{1}{p^{2}+\left(\frac{1}{\tau_{1}}+\frac{1}{\tau_{2}}\right) p+\frac{1}{\tau_{0}^{2}}+\frac{1}{\tau_{1} \tau_{2}}}\right) \Phi_{0}
$$

soit : $i_{\mathrm{L}^{\prime}}=F(p) . \Phi_{0}$.

La fonction de transfert précédente $F(p)$ est la plus générale possible de notre système.

Le flux de correction $\Phi_{c}$ traversant les bobines de lecture est lié au courant $i_{\mathrm{L}^{\prime}}$ des bobines de correction par $M_{0}$, le coefficient d'induction mutuelle des bobines de lecture et de correction : $\Phi_{\mathrm{c}}=M_{0} F(p) \Phi_{0}$.

La fonction de transfert en boucle ouverte (chaîne découplée virtuellement et totalement entre les bobines de lecture et de correction) est alors : $M_{0} . F(p)$.

Si l'on convient de prendre $\Phi_{0}$ positif, le flux de correction $\Phi_{c}$ doit être de signe opposé puisque la stabilisation doit provoquer une diminution du flux perturbateur, on en conclut que $M_{0} . F(p)$ doit être négatif. Ge résultat est obtenu en choisissant pour $M_{0}$ le signe convenable. Dans la pratique, un signe incorrect de $M_{0}$ se traduit par un accrochage du système; pour rétablir un fonctionnement correct, il suffit soit d'inverser les fils d'amenée du courant sur les bobines de correction, soit de retourner ces dernières face pour face.

e) Étude de la boucle d'asservissement en circuit fermé. La force électromotrice $E_{0}$ est fonction du flux $\Phi_{0}$, dû aux actions extérieures, qui traverse les bobines de lecture. Le système d'asservissement qui lui est adjoint constitue avant tout un système correcteur de flux et indirectement un système stabilisateur de champ magnétique.

Dans le cas où l'on utilise un système classique d'intégration dans la chaîne, on se reportera à l'étude faite par Fric [3] dans sa thèse. Nous allons montrer que dans notre montage utilisant un système d'intégration irréversible par lampe-photodiode, on obtient un asservissement plus simple (à retour unitaire) qu'avec les intégrateurs classiques. Nous définissons les différents flux :

$\Phi_{31}$ : flux traversant les bobines de lecture, dû aux bobines de l'électroaimant,

$\Phi_{21}$ : flux traversant les bobines de lecture, dû aux bobines de correction $\left(\Phi_{\mathrm{c}} \neq \Phi_{21}\right.$ car la chaîne n'est plus ouverte maintenant),
$\Phi_{32}$ : flux traversant les bobines de correction, dû aux bobines de l'électroaimant,

$\varphi_{0}$ : flux traversant les bobines de correction, dû à toutes les actions extérieures,

$\Phi_{12}$ : flux traversant les bobines de correction, dû aux bobines de lecture.

Nous supposerons que toutes les lignes de force du champ magnétique créé par l'électroaimant restent à l'intérieur du fer, ce qui entraîne : $\Phi_{31}=\Phi_{32}$; on a par ailleurs :

$\Phi_{0}=\Phi_{31}+\Phi_{21} \quad$ et $\quad \varphi_{0}=\Phi_{32}+\Phi_{12}=\Phi_{31}+\Phi_{12}$.

En toute rigueur, on a aussi :

$$
\Phi_{21}=M_{0}\left(i_{L^{\prime}}+i_{\varphi_{0}}\right)
$$

$i_{\varphi_{0}}$ est le courant créé dans les bobines de correction par les bobines de lecture et celles de l'électroaimant, courant dû au fait que nous avons fermé la boucle d'asservissement. Ce courant ne peut circuler qu'à travers les capacités parasites des bobines de correction, la photodiode présentant une résistance quasi infinie.

Ce courant est facilement calculable et a pour expression :

$$
\begin{gathered}
i_{\varphi_{0}}=-\frac{C^{\prime} N^{\prime} p^{2} \varphi_{0}}{\tau_{0}^{\prime} p^{2}+\tau^{\prime} p+1}=\psi(p) \varphi_{0} \\
\Phi_{21}=\Phi_{0}-\Phi_{31}=M_{0}\left[F(p) \Phi_{0}+\psi(p) \varphi_{0}\right] \\
\Phi_{0}-\Phi_{31}=M_{0} F(p) \Phi_{0}+M_{0} \psi(p)\left[\Phi_{31}+\Phi_{12}\right]
\end{gathered}
$$

mais :

$$
\Phi_{12}=M_{0} i_{\varphi_{0}}=M_{0} \varphi(p) \Phi_{0}
$$

d'où le rapport :

$$
\frac{\Phi_{0}}{\Phi_{31}}=\frac{1+M_{0} \psi(p)}{1-M_{0} F(p)-M_{0}^{2} \psi(p) \varphi(p)}
$$

Cette expression montre que dans le cas général le système n'est pas à retour unitaire. 
Dans la pratique, $i_{\varphi_{0}}$ est négligeable, ce qui entraîne $\psi(p)=0$ et :

$$
\frac{\Phi_{0}}{\Phi_{31}}=\frac{1}{1-M_{0} F(p)}
$$

expression caractéristique d'un retour unitaire, $M_{0} \cdot F(p)$ étant, comme nous l'avons dit précédemment, la fonction de transfert en circuit ouvert.

Ce dispositif permet en définitive de découpler complètement la chaîne de retour de l'entrée; alors que le système classique couple au maximum les bobines de lecture et de correction, notre dispositif les découple totalement dans le sens bobine de lecture $\rightarrow$ bobine de correction, c'est un couplage univoque.

Ce mode de fonctionnement qui, a priori, semblait impossible à obtenir avec deux bobines couplées devient en fait réalisable en veillant toutefois, lors de la construction des bobines de correction, à ce que ces dernières présentent une capacité parasite aussi faible que possible.

IV. Interprétation des résultats expérimentaux. a) Caractéristiques d'amplitude et de phase. - La caractéristique d'amplitude nous permet de connaître le gain en courant de tout le système lorsque les bobines de correction sont remplacées par une résistance.

Pour chaque fréquence, on a relevé la valeur du courant de correction en fonction de celle du courant d'alimentation des bobines magnétisantes de l'électroaimant, puis on a tracé la courbe donnant leur rapport $g$ en fonction de la fréquence ( fig. 7).

Compte tenu de la phase, cette courbe donne en fait l'efficacité du stabilisateur. Son allure se retrouvera lors de l'étude du coefficient de stabilisation en fonction de la fréquence.

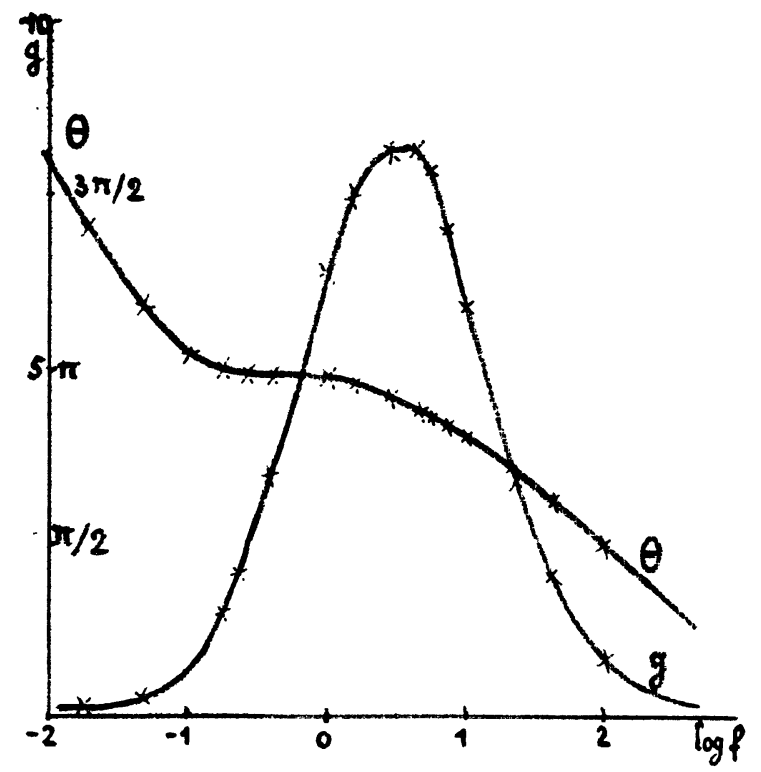

FIG. 7. - Courant $g$ et phase en fonction de la fréquence.
Le stabilisateur étant en fonctionnement normal, on a étudié aussi la phase $\theta$ du courant de correction par rapport à celle du courant d'alimentation des bobines de l'électroaimant ( fig. 7).

En adoptant comme sens positif le sens de parcours du courant magnétisant de l'électroaimant, on voit que l'efficacité de l'ensemble n'est effective pour la phase qu'entre $\pi / 2$ et $3 \pi / 2$, ce qui donne une bande passante comprise entre $1 / 100 \mathrm{de} \mathrm{Hz}$ et $200 \mathrm{~Hz}$.

b) Étude du coefficient de stabilisation. - Dans toute cette étude, nous avons toujours introduit une perturbation sinusoïdale de fréquence variable sur les bobines de l'électroaimant.

Dans une première expérience, nous avons mesuré simultanément la tension $V_{1}$ à la sortie de l'amplificateur (système en boucle ouverte : bobines de correction débranchées) et la tension $V_{2}$ à la sortie de la bobine placée au centre de l'entrefer.

Dans une deuxième expérience, nous avons mesuré les mêmes tensions $V_{1}^{\prime}$ et $V_{2}^{\prime}$ en boucle fermée. On a défini le coefficient de stabilisation interne par le rapport $\frac{V_{1}}{V_{1}^{\prime}}=K_{\mathrm{f}}$, c'est celui qui se déduit des flux précédents comme étant le rapport du flux perturbateur au flux résiduel.

De la même manière, nous avons défini le coefficient

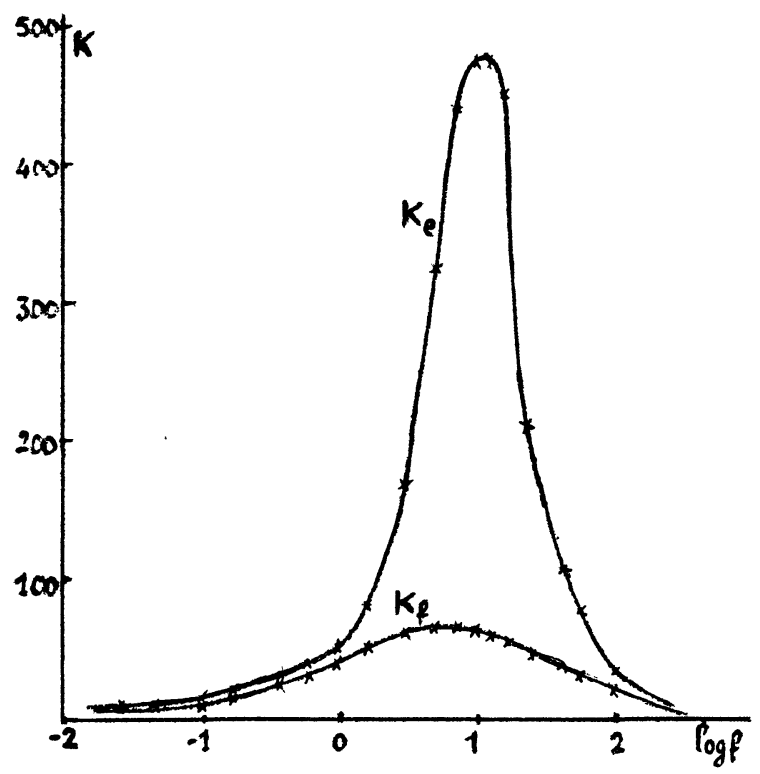

FIG. 8. - Coefficients de stabilisation au centre $\left(K_{\mathrm{e}}\right)$ et interne $\left(K_{\mathrm{f}}\right)$.

de stabilisation dans l'entrefer, pour la bobine utilisée, par le rapport : $\frac{V_{2}}{V_{2}^{\prime}}=K_{\mathrm{e}}$.

Les coefficients de stabilisation ainsi mesurés sont très différents l'un de l'autre, ainsi que le montrent les courbes expérimentales ( $f g .8$ ). 
Pour donner une explication à ce résultat, nous considérons qu'un tube de force, de petites dimensions autour de l'axe des pôles dans lequel s'écoule le flux créé par les bobines magnétisantes de l'électroaimant, présente une section constante $\Delta S$ dans le fer et dans l'air (fig. 9 a).

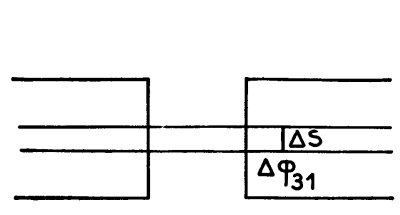

(a)

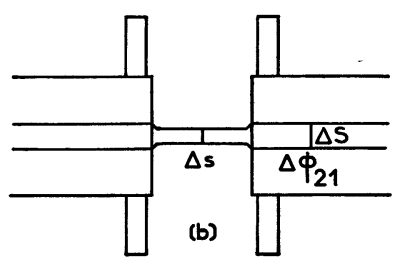

FIG. 9. - Lignes de champ dans l'entrefer.
Ce tube de force sera traversé par la variation de flux $\Delta \Phi_{31}$ correspondant au champ perturbateur.

Nous avons montré par ailleurs que les bobines de correction se comportaient comme des bobines de Helmoltz placées dans l'air.

On peut alors admettre que, pour ces dernières, la surface de la section droite d'un tube de force conservatif n'est pas la même dans le fer que dans l'air.

Cette surface sera $\Delta S$ dans le fer et $\Delta s$ au centre de l'entrefer (fig. $9 \mathrm{~b}$ ). Nous appelons $k$ le rapport $\Delta S / \Delta s$. Dans ce tube de force s'écoule le flux $\Delta \Phi_{21}$ créé par les bobines de correction.

On pourrait faire un raisonnement analogue pour les bobines de lecture et poser, pour le tube de force dans lequel s'écoule le flux $\Delta \Phi_{11}$ créé par les bobines de lecture :

$$
k^{\prime}=\Delta S / \Delta s^{\prime}
$$

Ce rapport définit une autre concentration, pas forcément égale à la précédente.

Il est alors possible de définir deux coefficients de stabilisation, l'un dans le fer $K_{\mathrm{f}}$ au niveau des bobines de lecture, l'autre $K_{\mathrm{e}}$ au centre de l'entrefer. Ils sont définis par les deux égalités suivantes :

$$
\begin{gathered}
K_{\mathrm{f}}=\frac{\Delta \Phi_{31}}{\Delta \Phi_{31}+\Delta \Phi_{21}+\Delta \Phi_{11}} \\
K_{\mathrm{e}}=\frac{\Delta \Phi_{31}}{\Delta \Phi_{31}+k^{\prime} \Delta \Phi_{11}+k \Delta \Phi_{21}}
\end{gathered}
$$

en précisant que $\Delta \Phi_{21}$ et $\Delta \Phi_{11}$ sont de signes opposés à $\Delta \Phi_{31}$.

Étant donné la forme très ramassée des bobines de lecture autour du pôle, on peut admettre en première approximation que $k^{\prime}=1$, ce qui donne pour $K_{\mathrm{e}}$ :

$$
K_{\mathrm{e}}=\frac{\Delta \Phi_{31}}{\Delta \tilde{\Phi}_{31}+\Delta \Phi_{11}+k \Delta \tilde{\Phi}_{21}}
$$

si nous admettons que la position des bobines de correction sur les pôles entraîne une concentration du flux créé par ces bobines, $k>1$, on en déduit immédiatement que $K_{\mathrm{e}}>K_{\mathrm{f}}$.

Ces résultats, envisagés pour un tube de force de section relativement petite, restent valables pour une section plus grande, par suite de la géométrie particulière des pôles, à condition toutefois que les lignes de force du champ magnétique restent perpendiculaires à la surface de la bobine réceptrice placée dans l'entrefer, la concentration restant uniforme.

S'il n'en est pas ainsi, le coefficient de stabilisation $K_{\mathrm{e}}$ dépend de la surface de la bobine.

On peut montrer que $K_{\mathrm{e}}$ peut devenir très grand par rapport à $K_{\mathrm{f}}$ malgré une très faible concentration du flux dans l'entrefer. Du point de vue logique, il ne saurait être question, en effet, d'admettre une forte concentration du flux dans un entrefer étroit.

Posons :

$$
\begin{aligned}
k & =1+\varepsilon \quad \varepsilon \text { étant petit devant } 1 \\
K_{\mathrm{e}} & =\frac{\Delta \Phi_{31}}{\Delta \Phi_{31}+\Delta \Phi_{11}+\Delta \Phi_{21}+\varepsilon \Delta \Phi_{21}} \\
\frac{1}{K_{\mathrm{e}}} & =\frac{1}{K_{\mathrm{f}}}+\varepsilon \frac{\Delta \Phi_{21}}{\Delta \Phi_{31}}
\end{aligned}
$$

mais :

$$
\frac{1}{K_{\mathrm{f}}}=1+\frac{\Delta \Phi_{21}}{\Delta \Phi_{31}}+\frac{\Delta \Phi_{11}}{\Delta \Phi_{31}} \quad \text { et } \quad \frac{\Delta \Phi_{21}}{\Delta \Phi_{31}} \simeq \frac{1}{K_{\mathrm{f}}}-1
$$

soit :

$$
K_{\mathrm{e}}=\frac{K_{\mathrm{f}}}{1-\varepsilon\left(K_{\mathrm{f}}-1\right)} .
$$

En général, le terme $\varepsilon\left(K_{\mathrm{f}}-1\right)$ est inférieur à 1 , mais il peut s'en rapprocher d'autant plus que $K_{\mathrm{f}}$ est plus grand et à la limite $K_{\mathrm{e}} \rightarrow \infty$. Rien ne s'oppose d'ailleurs à ce que $\varepsilon\left(K_{\mathrm{f}}-1\right)$ devienne supérieur à $1:$ il y a alors surcompensation.

On a pu vérifier par l'expérience l'ensemble des conclusions précédentes.

Conclusion. - On a montré qu'un dispositif d'asservissement utilisant un phénomène d'intégration par lampe permettait de découpler totalement le circuit des bobines de correction du reste du montage, le dispositif lampe-photodiode étant irréversible.

Par ailleurs, ce qui est un autre avantage, la photodiode se comporte comme un injecteur de courant, la phase de ce dernier étant indépendante des caractéristiques électriques de l'enroulement dans lequel il circule. 
L'ensemble constitue un stabilisateur de flux et, ou du champ pouvait être meilleure au centre de par extension, un stabilisateur de champ, l'un et l'entrefer qu'au niveau des bobines de lecture.

l'autre étant liés directement.

On a montré finalement que la stabilisation du flux

Manuscrit reçu le 5 novembre 1965.

\section{BIBLIOGRAPHIE}

[1] Raoult (G.), Adamski (J.), Cuemile (J.-P.), Mlle LaVAL (C.) et Roux (M.-R.), J. Physique Rad., 1962, 23, $47 \mathrm{~A}$.

[2] LavaI (C.), Diplôme d'Études Supérieures, ClermontFerrand, 20 juin 1961.

[3] FrIC (C.), Thèse d'État, Paris, décembre 1960.
[4] Blanc (F.), Fanguin (R.) et Raouli (G.), Brevet num. provisoire 992322, déposé le 22 octobre 1964.

[5] ANDREW (S. S.), Audio, décembre 1964, Mineola, N.Y. (U.S.A.).

[6] GUILLIEN (R.), Électronique, Presses Universitaires de France, 1961, p. 179. 